\title{
The quest for blue supergiants: Evolution of binary merger progenitors of Type-II peculiar supernovae and SN 1987A
}

\author{
Athira Menon and Alexander Heger \\ Monash Centre for Astrophysics (MoCA), \\ c/o School of Physics and Astronomy, Building 19, Monash University, Victoria, 3800, \\ Australia \\ email: athira.menon@monash.edu, alexander.heger@monash.edu
}

\begin{abstract}
We construct stellar evolution models until core collapse using KEPLER (Woosley \& Heger (2007)) to reproduce the observed signatures of the blue supergiant (BSG) progenitor of SN 1987A. This is based on the binary merger scenario proposed by Podsiadlowski (1992) and Ivanova et al. (2002). Various combinations of initial parameters for the binary components $\left(M_{1}=16-18 \mathrm{M}_{\odot}\right.$ and $\left.M_{2}=5-10 \mathrm{M}_{\odot}\right)$ and their merging, successfully match the He, N/C and $\mathrm{N} / \mathrm{O}$ ratios, along with the luminosity and effective temperature of the progenitor. Most of our models end their lives as BSGs. Thus we may be able to explain the origin of all Type IIP SNe that resemble SN 1987A through such mergers. We are currently working on the light curves and nuclear yields from the explosion of these models to compare them SN 1987A.
\end{abstract}

Keywords. SN 1987A, Binary mergers, Type IIP SNe

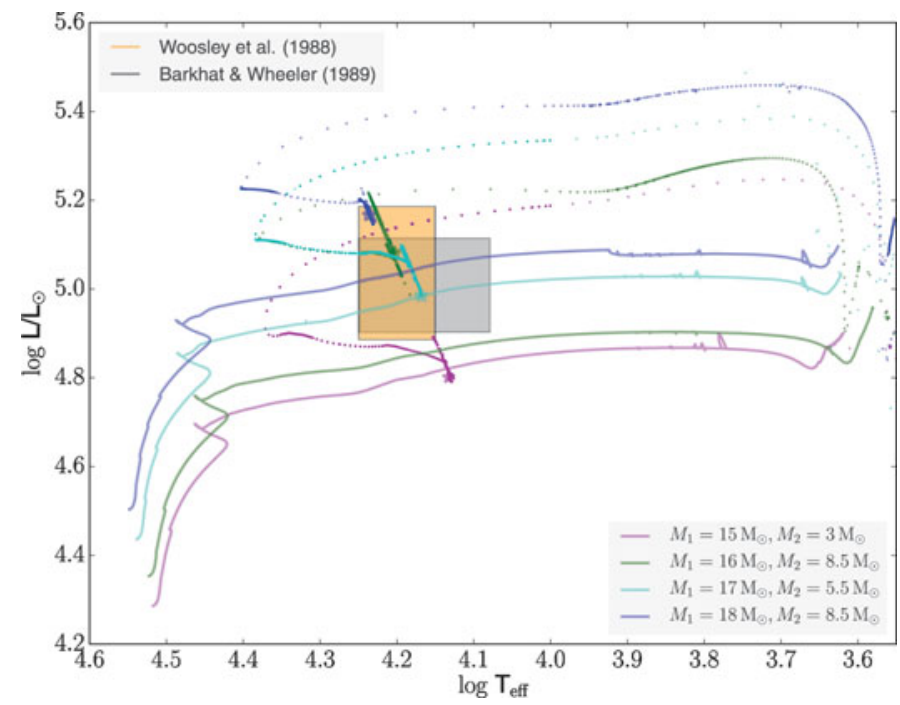

Figure 1. Evolutionary tracks of models with different initial masses $\left(M_{1}\right.$ and $\left.M_{2}\right)$. Final models ( $\star$ symbols) that lie in the shaded region are progenitor candidates for SN 1987A.

\section{References}

Ivanova, N., Podsiadlowski, P., \& Spruit, H. 2002, MNRAS, 334, 819

Podsiadlowski, P. 1992, PASP, 104, 717

Woosley, S. E. \& Heger, A., 2007, Physrep, 442, 269 